\title{
An infectious clone of the highly pathogenic porcine reproductive and respiratory syndrome virus: Topology of glycoprotein 3 (GP3) addressing the intrachain disulfide bonds
}

\author{
FENG Chun Yan ${ }^{1,2}$, LIU YueHuan ${ }^{3}$, YAN JingHua ${ }^{1} \&$ George F. Gao ${ }^{1,2,4^{*}}$ \\ ${ }^{1}$ CAS Key Laboratory of Pathogenic Microbiology and Immunology (CASPMI), Institute of Microbiology, Chinese Academy of Sciences, Beijing \\ 100101, China; \\ ${ }^{2}$ Graduate University of Chinese Academy of Sciences, Beijing 100049, China; \\ ${ }^{3}$ Institute of Animal Husbandry and Veterinary Medicine, Beijing Academy of Agriculture and Forestry Sciences, Beijing 100097, China; \\ ${ }^{4}$ Beijing Institutes of Life Science, Chinese Academy of Sciences, Beijing 100101, China
}

Received April 26, 2011; accepted June 9, 2011

\begin{abstract}
The highly pathogenic porcine reproductive and respiratory virus (hpPRRSV) with discontinuous 30 amino acid (aa) deletion as a gene marker has caused great economic loss in pig industry and since 2007 has become the dominant strain prevalent in China and Vietnam since 2007. Reverse genetics method is a powerful tool urgently needed to be used on the hpPRRSV to study the intriguing molecular mechanism of transcription, replication and the virulence determinant factors. In our study, we successfully constructed a full- length infectious clone, prBJSY07, based on hpPRRSV isolate, BJSY07. The rescued virus, vrBJSY07, showed similar growth characters to those of the parental virus, BJSY07. We also found that a rescued virus vBJSY07 generated from pBJSY07 was viable but displayed decreased and delayed reproductive capacity, which might be caused by two amino acids mutations, S83C and S117C in glycoprotein 3 (GP3), acquired during the preparation of the infectious clone. The topology of the wild type GP3 and the mutant were further analyzed by bioinformatics and revealed that the mutated GP3 possessed slightly altered structure, most likely by forming a new disulfide bond between the two new cysteine residues. As GP3 is a cysteine-rich glycoprotein, common for viral glycoproteins, our results show that GP3 can accommodate even more cysteine mutations. Based on this, a topological model of GP3 is proposed by addressing the intrachain disulfides.
\end{abstract}

porcine reproductive and respiratory syndrome virus, reverse genetic methods, infectious clone, glycoprotein 3, topology

Citation: $\quad$ Feng C Y, Liu Y H, Yan J H, et al. An infectious clone of the highly pathogenic porcine reproductive and respiratory syndrome virus: Topology of glycoprotein 3 (GP3) addressing the intrachain disulfide bonds. Chinese Sci Bull, 2011, 56: 2785-2793, doi: 10.1007/s11434-011-4631-8

Porcine reproductive and respiratory syndrome virus (PRRSV) causes reproductive failure and respiratory distress in pigs and results in tremendous economic losses in the pig industry worldwide [1-3]. The PRRSV is a member of Arteriviridae family of viruses, which are positivelystranded RNA viruses that include equine arteritis virus (EAV), lactate dehydrogenase-elevation virus (LDV), and simian hemorrhagic fever virus (SHFV) [4]. Like other members of this family, the genome of PRRSV consists of a linear, single RNA molecule approximately $15 \mathrm{~kb}$ in size

*Corresponding author (email: gaof@im.ac.cn) and encodes nine open reading frames (ORFs) flanked by untranslated regions (UTRs) at the $5^{\prime}$ - and 3 '-terminal $[5,6]$.

ORF $1 a$ and $O R F 1 b$ are located directly downstream of the $5^{\prime}$ leader and occupy more than two-thirds of the entire genome. These genes encode two non-structural polyproteins which can be further autoproteolytically cleaved into 13 smaller non-structural proteins involved in viral replication and transcription [7]. ORFs 2-7 are translated from a nested set of subgenomic RNA (sgRNA) encoding the structural proteins [8], including 5 envelope glycoproteins named GP2a (ORF2a), GP2b (ORF2b), GP3 (ORF3), GP4 (ORF4), and GP5 (ORF5), as well as nonglycosylated 
membrane protein $\mathrm{M}$ (ORF6), and the nucleocapsid protein $\mathrm{N}$ (ORF7) [1]. Based on the protein quantities in mature virions, these structural proteins are divided into 2 groups, major proteins and minor proteins. The former group includes M, N, and GP5 proteins, while the later includes the GP2a, GP2b, GP3 and GP4 [9].

In the summer of 2006, a highly pathogenic PRRSV (hpPRRSV) emerged and swept over half of the provinces of China, causing enormous economic losses. As reported previously, this virus is different from the typical PRRSV, with the clinical symptoms characterized by prolonged high fever $>41^{\circ} \mathrm{C}$, anorexia, red discoloration of the ears and body, desiccated excrement in the early period, and loose bowels in the later periods $[10,11]$. Further studies suggest that hpPRRSV possesses a discontinuous deletion of 30 aa in the non-structural protein 2 (NSP2) as a gene marker [12]. However, the molecular determinant of virulence or the function of each virus protein is still poorly understood. Further studies are urgently needed to explore the underlying molecular mechanism of pathogenesis and to develop a new generation vaccine.

In recent years, reverse genetic techniques have been extensively applied in the molecular genetic manipulation of RNA viruses, including hepatitis $C$ virus [13], dengue virus [14], and enterovirus 6 [15]. This method is very powerful for the analysis of gene expression [16], replication [17], function of viral proteins [18], recombination of RNA viruses, and pathogenesis by mutating individual amino acids [19], deleting coding regions [17], inserting foreign sequences [20], and generating chimeric virions [21]. It can also potentially be used to develop new genetically-modified vaccines [22]. Thus, establishing an infectious viral genomic clone would be extremely useful to investigate the biology of hpPRRSV. Although two similar infectious clones of the hpPRRSV isolates have been constructed and characterized recently, and the 30 discontinuous amino acid deletion was ruled out as being responsible for the high virulence [23,24], there are still many important scientific questions remaining to be answered. Therefore, the establishment of hpPRRSV infectious clones derived from different strains will provide some new tools for future pathogenesis studies.

In our study, BJSY07 (an hpPRRSV strain isolated from Beijing) was sequenced and chosen as a parental virus to construct two full-length infectious clones, pBJSY07 and prBJSY07. Reverse transcription polymerase chain reaction (RT-PCR) and immunofluorescence assays confirmed that two viruses named vBJSY07 and vrBJSY07 were rescued from the plasmid, respectively. In vrBJSY07, the cDNA sequence was the same as the wild-type virus, BJSY07, while in vBJSY07, two mutations, S83C and S117C, were found in the GP3 protein. Growth kinetics of the two rescued viruses suggested that vBJSY07 displayed decreased replicative ability than vrBJSY07. Further bioinformatics analysis of the topologies in the wild-type GP3 and mutated
GP3 revealed that two disulfide bonds might be mismatched and an extra disulfide bond was formed in mutated GP3. The slight topological alteration might be responsible for vBJSY07 having decreased reproducibility and also indicates that the virus could survive with extra disulfide mutation in GP3. Our study not only offers a successful reverse genetic platform for hpPRRSV, but also provided some clues for the molecular function of a virus structural protein, GP3.

\section{Materials and methods}

\subsection{Viruses and cells}

The PRRSV strain, BJSY07, isolated in 2007 from the Shunyi District of Beijing, China, was used as the parental virus in our study. Passage 7 of this strain was used to perform full-length genome sequencing. The complete genomic sequence was submitted to the GenBank database (GenBank accession: HM011104). Virus titers (expressed as $50 \%$ tissue culture infective dose $\left[\mathrm{TCID}_{50}\right]$ per milliliter) were determined on Marc-145 cells by end-point dilution, as described previously [25]. Marc-145 and 293T cells were cultured at $37^{\circ} \mathrm{C}$ with $5 \% \mathrm{CO}_{2}$ in DMEM medium (Invitrogen, Auckland, NY, USA) supplemented with $10 \%$ fetal bovine serum (HyClone Laboratories., South Logan, UT, USA).

\subsection{RNA isolation, RT-PCR, and sequence analysis}

Viral RNA was isolated from Marc-145 cells culture supernatant using Viral RNA Mini Kit (QIAGEN, USA). RTPCR was performed to amplify the full-length cDNA using sets of specific primers for hpPRRSV, as described previously [26]. The full-length genome was divided into 18 overlapping cDNA segments. Subsequently, the T-vector strategy was used to generate 18 recombinant plasmids for sequencing. This method was also used for confirmation of the rescued virus, vBJSY07, and the successful reverse mutation of vrBJSY07.

\subsection{Construction of full-length cDNA clones from BJSY07}

The full-length cDNA clone BJSY07 was constructed using the low copy number plasmid, pWSK29, by several subcloning steps, using the restriction sites present in the virus genome. The 5' primer contained T7 RNA polymerase promoter and a Cla I restriction enzyme site, the $3^{\prime}$ primer contained a 40 nucleotide (nt) poly(A) and an extra Not I enzyme site (Table 1). To distinguish the rescued virus from its parental sequence, a unique restriction enzyme site $S c a$ I was introduced into the ORF la coding region with a silent mutation, using PCR-based mutagenesis (Table 1). As a result, a substitution mutation ( $\mathrm{C}$ to $\mathrm{T}$ ) was created at 
Table 1 The primers used in the development of full-length cDNA clone construction

\begin{tabular}{llcl}
\hline \multicolumn{1}{c}{ Primer } & \multicolumn{1}{c}{ Sequence $\left(5^{\prime} \rightarrow 3^{\prime}\right)$} & Position & Use \\
\hline $5^{\prime}$ primer & AGGGCCATCGATTAATACGACTCACTATAGATGACGTATAGGTGTTGGCT & $1-20$ & Amplification \\
$3^{\prime}$ primer & GAATGCGGCCGCT 40 & $15344-15352$ & Amplification \\
$1881 \mathrm{~F}$ & TGCCTAGCAGTACTATCCC & $1867-1886$ & Engineered restriction sites \\
$1881 \mathrm{R}$ & GGGATAGTACTGCTAGGCA & $1867-1886$ & Engineered restriction sites \\
\hline
\end{tabular}

nucleotides position 1881. In this way, two full-length cDNA clones, named prBJSY07 (with the same sequence as wild-type virus) and pBJSY07 were obtained during the preparation of the infectious clones.

\subsection{In vitro transcription and transfection}

The plasmids, prBJSY07 and pBJSY07, were amplified by transformation to Escherichia coli $\mathrm{DH} 5 \alpha$ and grown at $28^{\circ} \mathrm{C}$, in the presence of $15 \mu \mathrm{g} / \mathrm{mL}$ of ampicillin to keep their copies as low as possible. The two plasmids were isolated using a QIAprep Spin Miniprep kit (QIAGEN) and linearized with the Not I restriction enzyme. Approximately $5 \mu \mathrm{g}$ of the purified linearized pBJSY07 was used as a template for in vitro transcription, using a $\mathrm{T} 7 \mathrm{mMessage}$ Machine Kit (Ambion, USA) according to the manufacturer's protocol [27]. The RNA was dissolved in nuclease-free water and purified with NucleoSpin RNAII kit (Macherey-Nagel, Germany). Subconfluent 293T cells were transfected with 2 $\mu \mathrm{g}$ RNA per well using the DMRIE-C reagent (Invitrogen) in six-well culture dishes. The medium was harvested $36 \mathrm{~h}$ after transfection and transferred to Marc-145 to rescue infectious virus. Cells were monitored daily for cytopathic effect (CPE).

\subsection{Immunofluorescence assay}

Marc-145 cells were grown in 24-well micro-plates with slides inside, as previously described [28], and were infected with $100 \mu \mathrm{L}, 10^{2} \mathrm{TCID}_{50} / \mathrm{mL}$ BJSY07, vBJSY07, and vrBJSY07, respectively. Uninfected Marc-145 cells were used as a control. After 24 or $48 \mathrm{~h}$, monoclonal antibodies (MAbs) directed against the $\mathrm{N}$ proteins were added to virus infected cells for staining. After incubation at room temperature for $2 \mathrm{~h}$, slides were washed three times with phosphate-buffered saline with $0.5 \%$ tween-20 (PBST), and fluorescein isothiocyanate (FITC)-conjugated anti-mouse IgG was added (1:1000 dilution). After $1 \mathrm{~h}$ of incubation, slides were washed three times, and observed under fluorescence microscope at $20 \times$ magnification.

\subsection{Growth curves of the viruses}

Marc-145 cells were seeded in 12-well plastic plates and inoculated at a multiplicity of infection (M.O.I.) of 1. The culture supernatant from infected cells were collected at the indicated time points $(24,48,72,96$, and $120 \mathrm{~h}$ post- infection), and stored at $-70^{\circ} \mathrm{C}$. Virus titration was performed by calculation of $\mathrm{TCID}_{50}$ and the titer was calculated according to the method of Reed and Muench [29]. To confirm these results, each experiment was repeated at least three times.

\subsection{Statistical analysis}

Data were expressed as means \pm standard deviations. Differences between repeats were compared with the use of the unpaired Student's $t$-test. All reported $P$-values were twosided and $P<0.05$ were considered statistically significant.

\section{Results}

\subsection{Construction and confirmation of the rescued virus vrBJSY07 and vBJSY07}

Using a multi-step cloning strategy, the full-length cDNA clone prBJSYO7 was constructed as summarized in Figure 1(a), using specified restriction enzymes of the virus. Fulllength sequencing revealed that prBJSY07 had an identical sequence to wild-type virus BJSY07. Another infectious clone, pBJSY07, with two mutations S83C and S117C in GP3 was also obtained by chance during the preparation of prBJSY07. To rescue infectious viruses vrBJSY07 and vBJSY07, 293T cells were transfected with the in vitrotranscribed RNA. We chose 293T cells to assemble the first passage virions for their high efficiency of RNA transfection; higher than BHK21 cells which had been successfully used in previous reports $[22,27,30]$. The harvested cell medium containing the rescued virus vrBJSY07 and vBJSY07 particles were then each transferred to PRRSV permissible cells.

To confirm the recovery of vrBJSY07 and vBJSY07, a specific 1.2-kb fragment containing the engineered Sca I site was amplified from cell culture supernatants by RTPCR (Figure 1(b)). Digestion with Sca I of this fragment and the chromatogram of the sequencing reaction revealed that the rescued viruses, vrBJSY07 and vBJSY07, contained the engineered Sca I site (Figure 1(b) and (c)). Therefore, we were able to exclude the possibility that the rescued viruses were contaminated with wild-type virus. Furthermore, the synthesis of hpPRRSV viral nucleoprotein was detected in Marc-145 cells $24 \mathrm{~h}$ post-infection (hpi) and 48 hpi by immunofluorescence assay using PRRSV-specific nucleoprotein monoclonal antibodies (Figures 2 and 3). Consistent 
(a) $5^{\prime}$ UTR

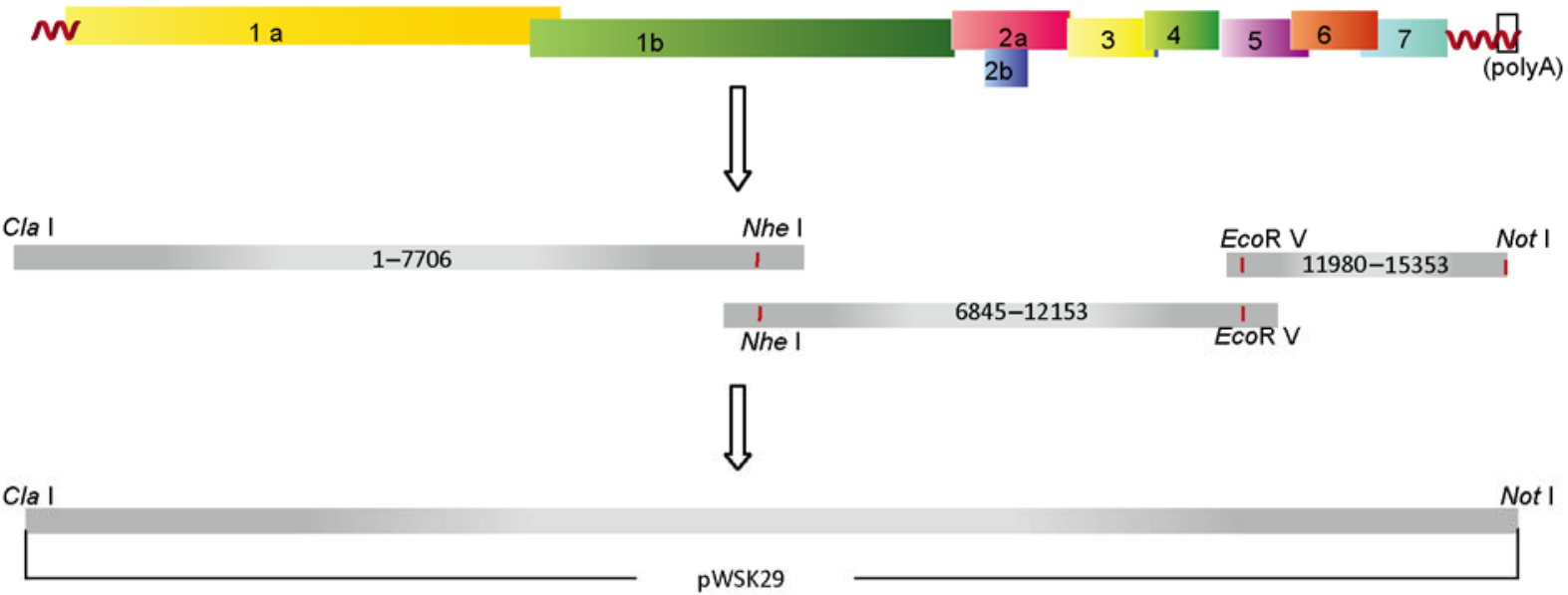

(b)

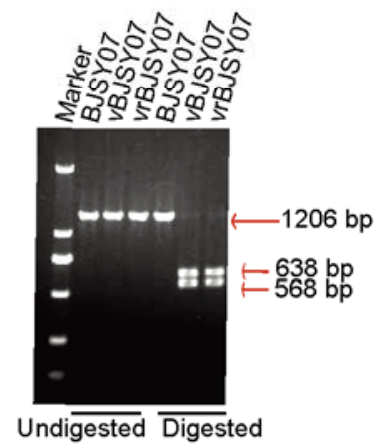

(c)

Figure 1 Schematic illustration of the construction strategy of genome-length cDNA clone and the identification of the engineered gene marker of rescued viruses. (a) The genome organization of BJSY07 is shown at the top of the figure and the colored boxes represent the different ORFs. Corresponding restriction sites used in the construction strategy are indicated. The full-length virus cDNA was finally cloned into a low-copy plasmid pWSK29 with $C l a$ I at 5' proximal and Not I at 3' proximal, downstream of poly(A). (b) Restriction endonuclease analysis of RT-PCR products from BJSY07, vBJSY07, and vrBJSY07. The results show that the engineered Sca I was detected in the vBJSY07 and vrBJSY07, but not detected in wild type BJSY07. (c) Chromatograms of the fragments containing the engineered Sca I site. The top shows the wild type BJSY07 which did not contain the enzyme site. The bottom shows the progeny virus which contained an engineered $S c a$ I site. The sequences of the parental and progeny viruses marked by red boxes indicate substitution from $\mathrm{C}$ to $\mathrm{T}$.
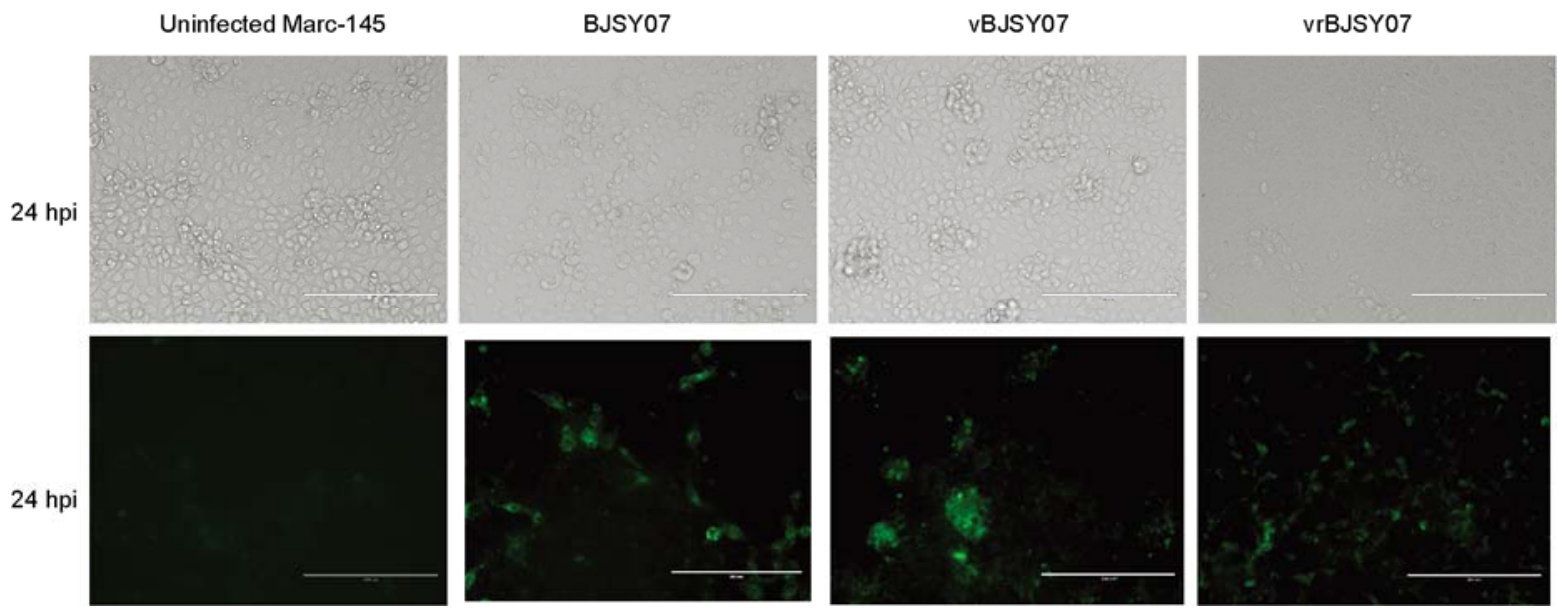

Figure 2 The detection of parental and rescued viruses by immunofluorescence assay at 24 hpi. Immunostaining was performed using a monoclonal antibody against nucleoprotein and photographs were taken under white and fluorescent light at $20 \times$ magnification. Bar scale $=200 \mu \mathrm{m}$. 

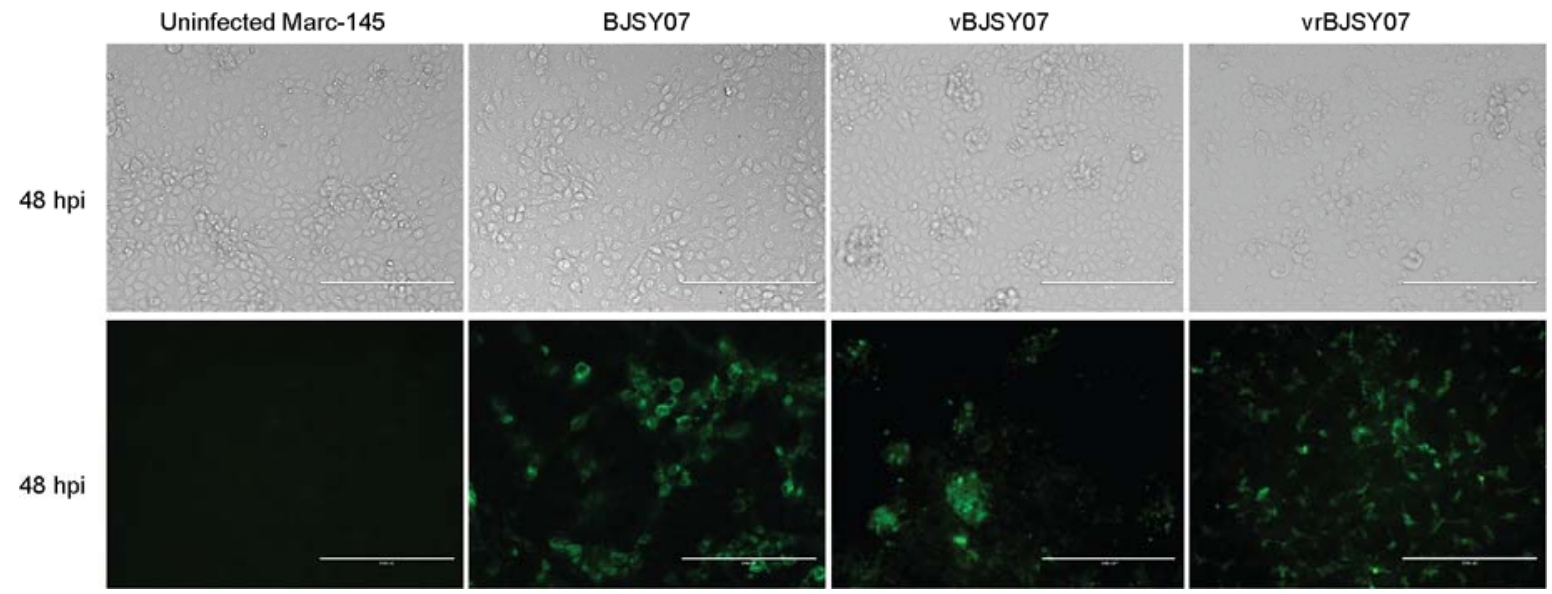

Figure 3 The observation of parental and rescued viruses by immunofluorescence assay at 48 hpi. Immunostaining was performed using a monoclonal antibody against nucleoprotein and photographs were collected under white and fluorescent light at $20 \times$ magnification. Bar scale $=200 \mu \mathrm{m}$.

with the RT-PCR results, nucleoprotein could be detected at 24 hpi and 48 hpi in Marc-145 cells infected with BJSY07, vrBJSY07 or vBJSY07. These results suggested that vrBJSY07 and vBJSY07 could replicate in Marc-145 cells at the similar levels as its parent, BJSY07.

\subsection{Comparison of the growth properties of different viruses}

The growth curves of BJSY07, vrBJSY07, and vBJSY07 were further determined. As shown in Figure 4, the peak of BJSY07 in growth curves was appeared at 72 hpi and the peak titer almost reached to $10^{7} \mathrm{TCID}_{50} / \mathrm{mL}$. The growth property of vrBJSY07 was indicated that the peak of growth curves appeared at $72 \mathrm{hpi}$ and the titer reached $10^{6.2}$ $\mathrm{TCID}_{50} / \mathrm{mL}$ (Figure 4). However, the peak of vBJSY07 appeared at $96 \mathrm{hpi}$ and the peak titer was just $10^{3} \mathrm{TCID}_{50} / \mathrm{mL}$ (Figure 4).

By comparing the growth curves of BJSY07, vBJSY07, and vrBJSY07, we found that vrBJSY07 had similar growth

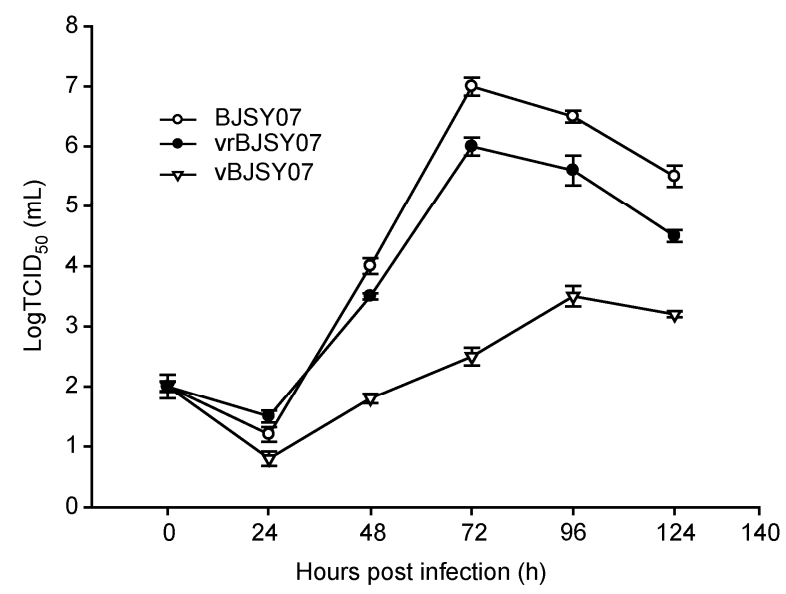

Figure 4 Comparison of the growth curves of wild-type virus (BJSY07) and rescued viruses (vBJSY07 and vrBJSY07). The $x$-axis indicates hours post infection (hpi) and the $y$-axis indicates the titer of the virus. curves to wild-type virus BJSY07, but was more efficiently reproduced than vBJSY07. As shown in Figure 4, the peak titer of vBJSY07 was much lower than vrBJSY07. In addition, the peak of vBJSY07 appeared at $96 \mathrm{hpi}$, showing an obvious delay not seen for BJSY07 and vrBJSY07, in which the first peak appeared at 72 hpi. Taken together, these results suggested that BJSY07 and vBJSY07 showed different growth kinetics. These results were also consistent with immunofluorescence against NP showed at $48 \mathrm{hpi}$, which the immunofluorescence indirectly reflected that the wildtype virus, BJSY07, has obviously more rapidly produced virus particles than vBJSY07 (Figures 2 and 3). The fragment containing the mutation sites was also amplified from vBJSY07 and vrBJSY07 by RT-PCR, and the sequencing results indicated that the mutations (S83C and S117C) existed in pBJSY07 could also be detected in vBJSY07, but were not found in vrBJSY07 (Figure 5). These results indicated that S83C and S117C mutations in GP3 might impair the virus particle production.

\subsection{Topological analysis of wild type GP3 and mutated GP3}

To analyze the influence of the mutations on the topology of GP3, the secondary structure and disulfide bonds were predicted using the SCRATCH protein predictor (http://www. ics.uci.edu/ baldig/scratch/). Transmembrane regions were also predicted using the TMHMM server (http://www.cbs. dtu.dk/services/TMHMM/), and two transmembrane regions were identified on the basis of our prediction results and previous report [31]. Models to reveal the interaction between the structural proteins were constructed based on reports [31,32] and our findings (Figure 6(a)) and the topological alteration of GP3 was constructed as shown in Figure 6(b) and (c). For wild type GP3, the secondary structure prediction showed that 106 aa out of 254 aa in GP3 formed coils in the structure, which indicated that GP3 might be very flexible especially in ectodomain of the protein. As a 


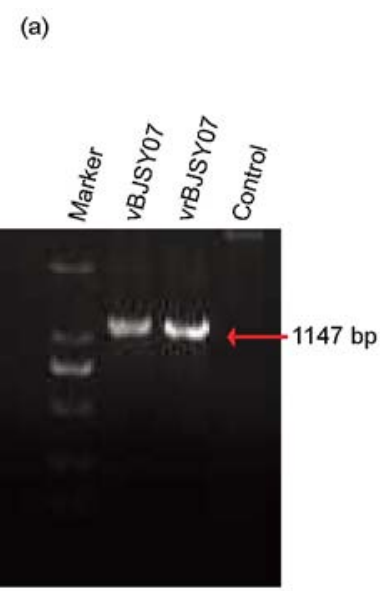

(b)

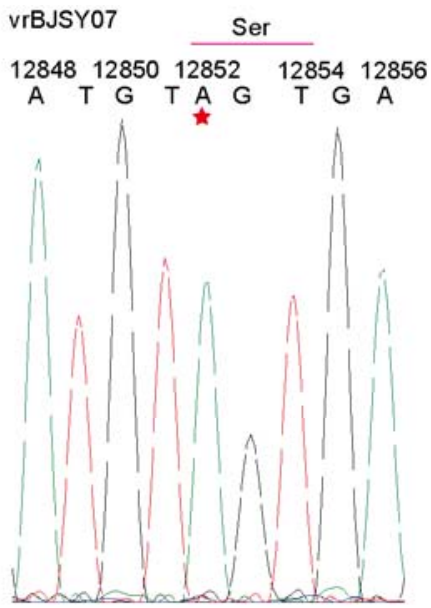

(d)

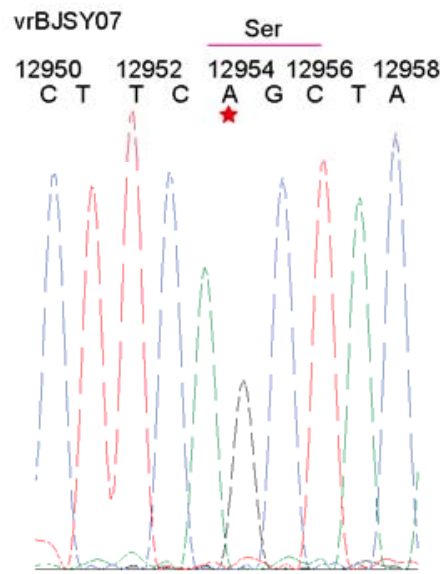

(c)

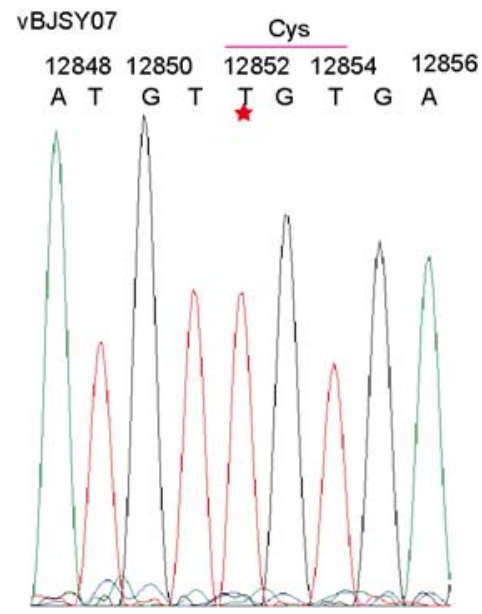

(e)

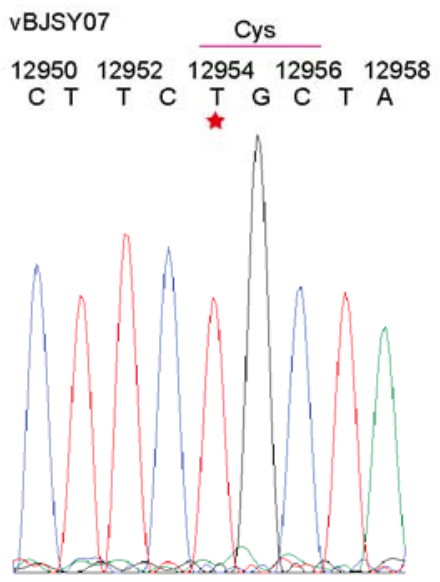

Figure 5 Confirmation of the mutations in vrBJSY07 and vBJSY07. (a) RT-PCR products from 12599 to 13717 nt of genome were amplified from vBJSY07 and vrBJSY07 indicated in the chromatograms Marc-145 cells were used as a control. The 1147-bp length fragments containing the mutation sites were then subcloned into the pGEM-T vector for sequencing. (b) The partial chromatogram of the RT-PCR fragment sequence from vrBJSY07. The mutation site A was marked by red star. (c) The partial chromatogram of the RT-PCR fragment sequence from vBJSY07. The mutated T was marked by red star, which resulted in S83C. (d) The partial chromatogram of the RT-PCR fragment from vrBJSY07. The mutation site A was marked by red star. (e) The partial chromatogram of the RT-PCR fragment from vBJSY07. The T mutation was marked by red star, which resulted in S117C. The numbers in the chromatogram were used to indicate the nucleotide positions in the genome.

cysteine rich protein, GP3 had 10 cysteine residues in the full-length protein. The positions of cysteine residues were C5 (cysteine in position 5), C14, C21, C22, C33, C54, C57, $\mathrm{C} 75, \mathrm{C} 82$ and $\mathrm{C} 143$. Eight cysteine residues were predicted to form four disulfide bonds, including disulfide bonds between $\mathrm{C} 5$ and $\mathrm{C} 22, \mathrm{C} 14$ and $\mathrm{C} 33, \mathrm{C} 57$ and C75, and C82 and C145. However, 5 cysteine residues including C54, C57, $\mathrm{C} 75, \mathrm{C} 82$, and $\mathrm{C} 143$ were predicted to be located in the ectodomain of GP3 and the remainder were predicted to be either located in the predicted transmembrane region or in the short intracellular region. Thus, the disulfide bonds between C5 and C22, C14 and C33 might not exist for the reason that they are located in the transmembrane domain. Only two disulfide bonds were probably formed between C57 and C75, C82 and C145 (Figure 1(b)). The C54 in wild type GP3 acted as a free cysteine. The prediction of mutated GP3 showed that the cysteine residues generated in position 83 and 117 formed an intrachain disulfide bond. More interestingly, the disulfide bonds between C57 and C75, $\mathrm{C} 82$, and $\mathrm{C} 145$ were predicted to be mismatched where they switched into the disulfide bonds between C54 and C75, C57 and C82 (Figure 6(c)); C145 instead of C54 in the mutated GP3 acted as a free cysteine. Thus, the prediction showed the mutations might slightly alter the topology of GP3 but would not affect the virus survival.

\section{Discussion}

With the rapid evolving capacity of RNA viruses, the hpPRRSV that emerged in China in 2006 shows significant differences from the previous viruses in the aspects of clinical symptoms and molecular characters [10,12]. Particularly, numerous adult pigs and some pregnant sows have died as a result of infection, while in the previous occurrences of PRRSV infection, most of the fatal cases had 
(a)

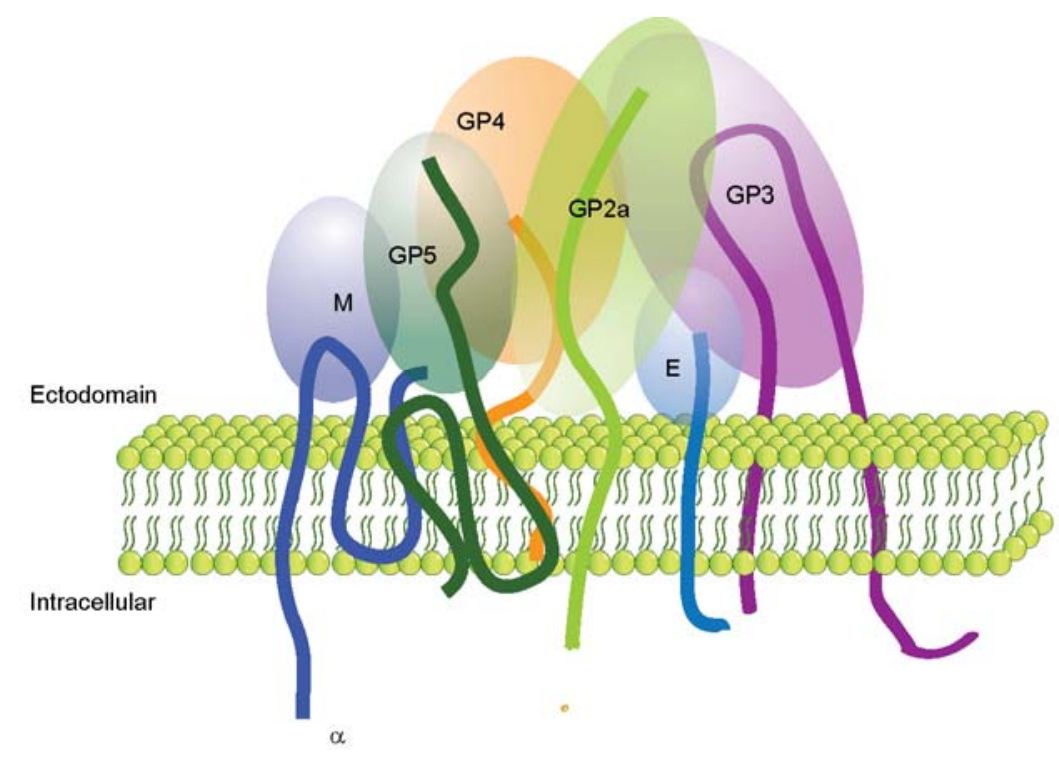

(b)

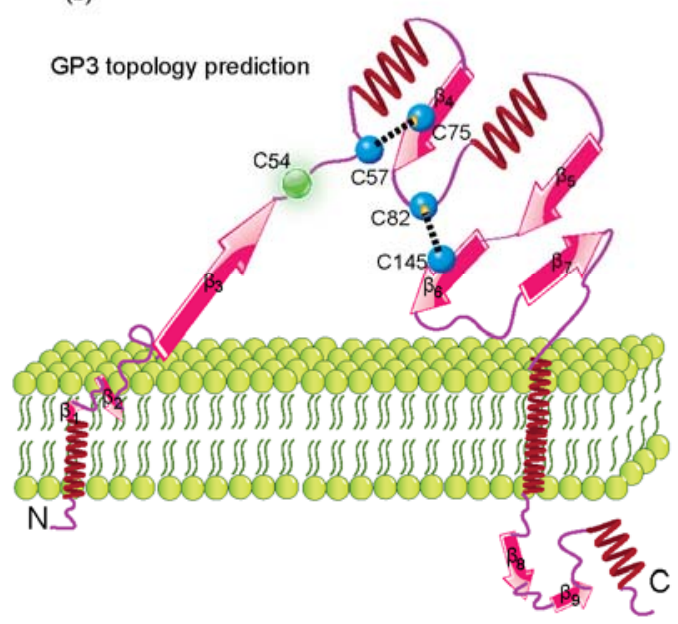

(c)

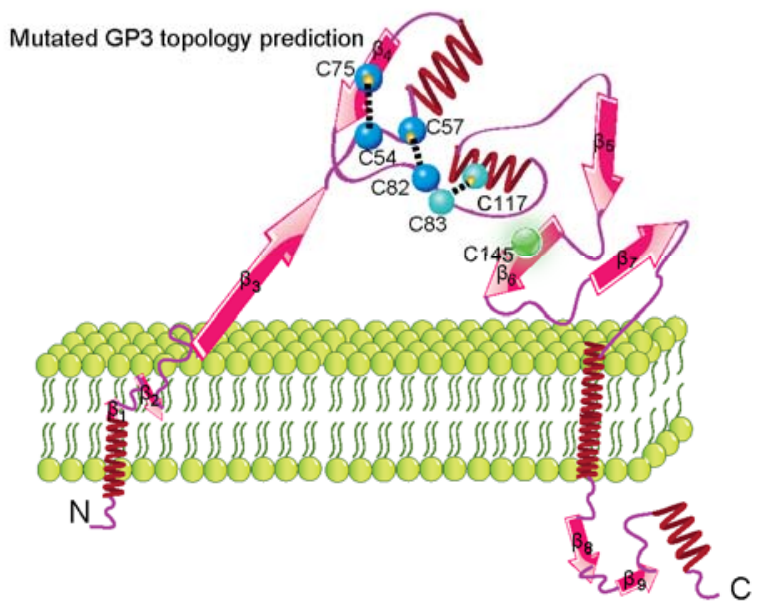

Figure 6 The interaction model for structural proteins in the surface-protein complex and the topology prediction of GP3. (a) The preliminary model of GP3 interacted with other structural proteins summarized according to references [31,32]. For the sake of simplicity and convenience, the GPs were depicted as globular structures with the same colored lines spanning the viral membrane. (b) The topology prediction of wild-type GP3, addressing its disulfide bonds. The secondary structure of the GP3 was indicated by curves, strands and helix. The black dashed line indicates the disulfide bond. The cysteine residues involved in the disulfide bonds are indicated by blue balls. Two disulfide bonds were predicted between C57 and C75, C82 and C145. The free cysteine C54 is indicated by a green ball. (c) The topology prediction of mutated GP3 with S83C and S117C. The cysteine residues involved in the disulfide bonds are indicated by blue balls, and the disulfide bonds are predicted to form between C54 and C75, C57, and C82. The mutated C83 and C117 are indicated by cyan balls. The free cysteine, C145, is indicated by a green ball.

occurred in nursery piglets. A reverse genetic platform based on hpPRRSV strain was urgently needed for the detailed molecular characterization of hpPRRSV and for the exploration of the new generation of vaccines.

In recent years, several PRRSV stains have been used as parental viruses to construct full-length infectious clones, including FL12, VR2332, P129, CH-1a, Lelystad virus, and the hpPRRSV JXwn06 and JX143 [18,23,24,27,28,30]. Although JXwn06 and JX143 as hpPRRSV were used to construct the infectious clones, the construction of a reverse genetic platform based on the hpPRRSV was still needed in a different strategy for easier screening and testing of new antiviral drugs in future studies. Thus, in our study, we constructed an infectious clone based on the other strain of hpPRRSV named BJSY07. During our construction, the infectious clone was based on 18 overlapping segments covering the full-length genome, so that further manipulation would be easier. Besides, 293T cells instead of BHK21 were used to guarantee the transfection efficiency of fulllength infectious RNA. Finally, the platform of the infectious clone was completed and two rescued viruses, vrBJSY07 and vBJSY07 were successfully rescued, confirmed by RT-PCR and immunofluorescence assays (Figures 1(b), 2, 3, and 5).

As a result, vrBJSY07 possessed similar growth properties to the wild-type virus, BJSY07 (Figure 4). Only minor 
biological differences were found between the two strains. The titer at the peak of BJSY07 replication was slightly higher than that of vrBJSY07. The same situation was also found in other reports, where the cloned virus was qualitatively similar but quantitatively slightly different from parental virus [30].

Of particular interest to us, vBJSY07 showed obviously decreased and delayed reproduction compared with vrBJSY07 (Figure 4). Two mutations in GP3, S83C and S117C in GP3 were demonstrated to be account for the decrease and delay in our study. Such kinds of decrease and delay also have been observed in the partly deletion of nsp2 in PRRSV, which could be explained by the deletion in nsp2 interfering the function of $n s p 2[17,33]$.

Until now, little is known yet about the functions of the minor glycoproteins GP3, except that it is required for generation of infectious virions [34]. As reported, GP3 might function collectively by interacting with GP2a and GP4 in virion as multimeric complex $[29,33,34]$. It also has been reported in EAV, GP3-GP2b/GP4 complex is disulfide linked when the virions leave the cells [34,35]. A model was constructed to reflect the interaction between the structural proteins according to previous reports, with our new observations (Figure 6(a)). However, no direct structural information of GP3 is yet available. The secondary structure prediction of GP3 in our study indicated that GP3 was very flexible in ectodomain, which might contribute to its multifunction in interaction with different structural proteins. Further disulfide bonds prediction indicated that the mutations resulted in an emergence of a potential intrachaindisulfide linkage, between C83 and C117 (Figure 6(c)), which would interfere with the original disulphide bonds in the wild type virus, resulting in the mismatch of the other two disulfide bonds. The disulfide bonds between C57 and $\mathrm{C} 75, \mathrm{C} 82$ and $\mathrm{C} 145$ were switched into the disulfide bonds between C53 and C75, C57 and C82. C143 instead of C54 was released as a free cysteine. Thus, the mutations might alter the topology of GP3, which influence the interaction with other structural protein in the multi-protein complex, and this influence was further resulted in the growth difference between vrBJSY07 and vBJSY07. Thus, the multiprotein complex of minor structural protein might play an important role in virus particle production. However, the overall topology might not be influenced by the mutation, which explained the viability but not lethality to vBJSY07. At this point, we could conclude that as a cysteine-rich protein, GP3 could accommodate more cysteine residues in its structure, which was unusual for a surface glycoprotein. This might also indicate that GP3 is very flexible and can accommodate different conformations in the surface-protein complex.

In conclusion, the emergence of hpPRRSV in China in 2006 has resulted in great economic losses. It is imperative to obtain reagents to uncover the intriguing molecular mechanism of these viruses. In our study, a reverse genetic platform was successfully constructed based on BJSY07, a representative isolate of hpPRRSV. Two progeny viruses named vrBJSY07 and vBJSY07 were been rescued and vrBJSY07 was shown to be genetically identical and showed similar growth kinetics with the wild-type virus BJSY07. By contrast, vBJSY07 showed decreased and delayed growth kinetics compared with vrBJSY07. The two mutations, S83C and S117C, in GP3 might form a new disulfide bond and resulted in mismatched disulfide bonds that might influence the topology of GP3 and resulted in the decreased reproductive capacity of vBJSY07. Taken together, based on our reverse genetic platform, further exploration of the functional research of virus proteins is available. This might in turn allow the production of a new generation of genetically engineered PRRSV vaccines.

We thank Ede Qing for providing the plasmid, pwsk29. We also thank Kegong Tian for the NP monoclonal antibody. This work was supported by the National High Technology Research and Development Program of China (2006BAD06A04) and the National Natural Science Foundation of China (80121003).

1 Meulenberg J J. PRRSV, the virus. Vet Res, 2000, 31: 11-21

2 Wensvoort G, Terpstra C, Pol J M, et al. Mystery swine disease in The Netherlands: The isolation of Lelystad virus. Vet Q, 1991, 13: $121-130$

3 Sur J H, Doster A R, Christian J S, et al. Porcine reproductive and respiratory syndrome virus replicates in testicular germ cells, alters spermatogenesis, and induces germ cell death by apoptosis. J Virol, 1997, 71: 9170-9179

4 Meulenberg J J, Hulst M M, de Meijer E J, et al. Lelystad virus, the causative agent of porcine epidemic abortion and respiratory syndrome (PEARS), is related to LDV and EAV. Virology, 1993, 192: $62-72$

5 Conzelmann K K, Visser N, van Woensel P, et al. Molecular characterization of porcine reproductive and respiratory syndrome virus, a member of the arterivirus group. Virology, 1993, 193: 329-339

6 Thiel H J, Meyers G, Stark R, et al. Molecular characterization of positive-strand RNA viruses: Pestiviruses and the porcine reproductive and respiratory syndrome virus (PRRSV). Arch Virol Suppl, 1993, 7: 41-52

7 van Dinten L C, Rensen S, Gorbalenya A E, et al. Proteolytic processing of the open reading frame $1 \mathrm{~b}$-encoded part of arterivirus replicase is mediated by nsp4 serine protease and Is essential for virus replication. J Virol, 1999, 73: 2027-2037

8 Dea S, Gagnon C A, Mardassi H, et al. Current knowledge on the structural proteins of porcine reproductive and respiratory syndrome (PRRS) virus: Comparison of the North American and European isolates. Arch Virol, 2000, 145: 659-688

9 Nelson E A, Christopher-Hennings J, Benfield D A. Structural proteins of porcine reproductive and respiratory syndrome virus (PRRSV). Adv Exp Med Biol, 1995, 380: 321-323

10 Tong G Z, Zhou Y J, Hao X F, et al. Highly pathogenic porcine reproductive and respiratory syndrome, China. Emerg Infect Dis, 2007, 13: $1434-1436$

11 Tian K, Yu X, Zhao T, et al. Emergence of fatal PRRSV variants: Unparalleled outbreaks of atypical PRRS in China and molecular dissection of the unique hallmark. PLoS One, 2007, 2: e526

12 Feng Y, Zhao T, Nguyen T, et al. Porcine respiratory and reproductive syndrome virus variants, Vietnam and China, 2007. Emerg Infect Dis, 2008, 14: 1774-1776

13 Hou W, Aoki C, Yu L, et al. A recombinant replication-competent hepatitis $\mathrm{C}$ virus expressing Azami-Green, a bright green-emitting fluorescent protein, suitable for visualization of infected cells. Bio- 
chem Biophys Res Commun, 2008, 377: 7-11

14 Zhu W, Qin C, Chen S, et al. Attenuated dengue 2 viruses with deletions in capsid protein derived from an infectious full-length cDNA clone. Virus Res, 2007, 126: 226-232

15 Blackburn R V, Racaniello V R, Righthand V F. Construction of an infectious cDNA clone of echovirus 6. Virus Res, 1992, 22: 71-78

16 Fang Y, Rowland R R, Roof M, et al. A full-length cDNA infectious clone of North American type 1 porcine reproductive and respiratory syndrome virus: Expression of green fluorescent protein in the Nsp2 region. J Virol, 2006, 80: 11447-11455

17 Han J, Liu G, Wang Y, et al. Identification of nonessential regions of the nsp2 replicase protein of porcine reproductive and respiratory syndrome virus strain VR-2332 for replication in cell culture. J Virol, 2007, 81: 9878-9890

18 Lee C, Calvert J G, Welch S K, et al. A DNA-launched reverse genetics system for porcine reproductive and respiratory syndrome virus reveals that homodimerization of the nucleocapsid protein is essential for virus infectivity. Virology, 2005, 331: 47-62

19 Ran Z G, Chen X Y, Guo X, et al. Recovery of viable porcine reproductive and respiratory syndrome virus from an infectious clone containing a partial deletion within the Nsp2-encoding region. Arch Virol, 2008, 153: 899-907

20 Kim D Y, Calvert J G, Chang K O, et al. Expression and stability of foreign tags inserted into nsp2 of porcine reproductive and respiratory syndrome virus (PRRSV). Virus Res, 2007, 128: 106-114

21 Huang Y W, Fang Y, Meng X J. Identification and characterization of a porcine monocytic cell line supporting porcine reproductive and respiratory syndrome virus (PRRSV) replication and progeny virion production by using an improved DNA-launched PRRSV reverse genetics system. Virus Res, 2009, 145: 1-8

22 Yoo D, Welch SK, Lee C, et al. Infectious cDNA clones of porcine reproductive and respiratory syndrome virus and their potential as vaccine vectors. Vet Immunol Immunopathol, 2004, 102: 143-154

23 Lv J, Zhang J, Sun Z, et al. An infectious cDNA clone of a highly pathogenic porcine reproductive and respiratory syndrome virus variant associated with porcine high fever syndrome. J Gen Virol, 2008, 89: 2075-2079

24 Zhou L, Zhang J, Zeng J, et al. The 30-amino-acid deletion in the
Nsp2 of highly pathogenic porcine reproductive and respiratory syndrome virus emerging in China is not related to its virulence. J Virol, 2009, 83: 5156-5167

25 Wensvoort G, Terpstra C, Boonstra J, et al. Production of monoclonal antibodies against swine fever virus and their use in laboratory diagnosis. Vet Microbiol, 1986, 12: 101-108

26 Sun Y, Xue F, Guo Y, et al. Crystal Structure of Porcine Reproductive and Respiratory Syndrome Virus (PRRSV) Leader Protease Nsp1 \{alpha\}. J Virol, 2009,83: 10931-10940

27 Meulenberg J J, Bos-de Ruijter J N, van de Graaf R, et al. Infectious transcripts from cloned genome-length cDNA of porcine reproductive and respiratory syndrome virus. J Virol, 1998, 72: 380-387

28 Truong H M, Lu Z, Kutish G F, et al. A highly pathogenic porcine reproductive and respiratory syndrome virus generated from an infectious cDNA clone retains the in vivo virulence and transmissibility properties of the parental virus. Virology, 2004, 325: 308-319

29 Reed L J, Muench H. A simple method of estimating fifty percent endpoints. Am J Hygiene, 1938, 27: 493-497

30 Nielsen H S, Liu G, Nielsen J, et al. Generation of an infectious clone of VR-2332, a highly virulent North American-type isolate of porcine reproductive and respiratory syndrome virus. J Virol, 2003, 77: 3702-3711

31 Dokland T. The structural biology of PRRSV. Virus Res, 2010, 154: 86-97

32 Das P B, Dinh P X, Ansari I H, et al. The minor envelope glycoproteins GP2a and GP4 of porcine reproductive and respiratory syndrome virus interact with the receptor CD163. J Virol, 2010, 84: $1731-1740$

33 Faaberg K S, Kehrli ME Jr, Lager K M, et al. In vivo growth of porcine reproductive and respiratory syndrome virus engineered nsp2 deletion mutants. Virus Res, 2010, 154: 77-85

34 Wissink E H, Kroese M V, van Wijk H A, et al. Envelope protein requirements for the assembly of infectious virions of porcine reproductive and respiratory syndrome virus. J Virol, 2005, 79: 1249512506

35 Wieringa R, de Vries A A, Rottier P J. Formation of disulfide-linked complexes between the three minor envelope glycoproteins (GP2b, GP3, and GP4) of equine arteritis virus. J Virol, 2003, 77: 6216-6226

Open Access This article is distributed under the terms of the Creative Commons Attribution License which permits any use, distribution, and reproduction in any medium, provided the original author(s) and source are credited. 